\title{
Welche Bedeutung hat die spezifische Serotonin-5-HT1F-Rezeptor Blockade bei einer akuten Migräneattacke
}

Ist ein spezifischer Serotonin-5HT1F-Rezeptoragonist ohne vasokonstriktive Eigenschaften bei der Behandlung akuter Migräneattacken wirksam? Erste Studiendaten sprechen dafür.

$\mathrm{D}$ ie Einführung der Triptane vor zwanzig Jahren war ein Durchbruch bei der Behandlung akuter Migräneattacken, insbesondere bei Patienten, die auf Analgetika oder nicht steroidale Antirheumatika (NSAR) nicht ansprachen. Triptane wirken auf Serotonin-5-HT1Bund -1D-Rezeptoren. Über 5-HT1BRezeptoren werden vasokonstriktive Ei- schaften aufweisen. Dazu gehören unter anderem die 5-HT1F-Rezeptoragonisten. 5-HT1F-Rezeptoren werden im Ganglion Gasseri des Nervus trigeminus und im Nucleus caudalis des Nervus trigeminus exprimiert. Lasmiditan ist ein hochselektiver 5-HT1F-Rezeptoragonist, der nicht an andere Serotonin-Rezeptoren bindet. Mit dieser Phase-II-Dosisfindungsstudie sollte belegt werden, dass Lasmiditan zur Behandlung akuter Migräneattacken geeignet ist.

Die Patienten wurden in fünf Gruppen randomisiert und erhielten intravenös 50, 100, 200 oder $400 \mathrm{mg}$ Lasmiditan oder Placebo. Der primäre Endpunkt war die Besserung der Kopfschmerzen von schwer

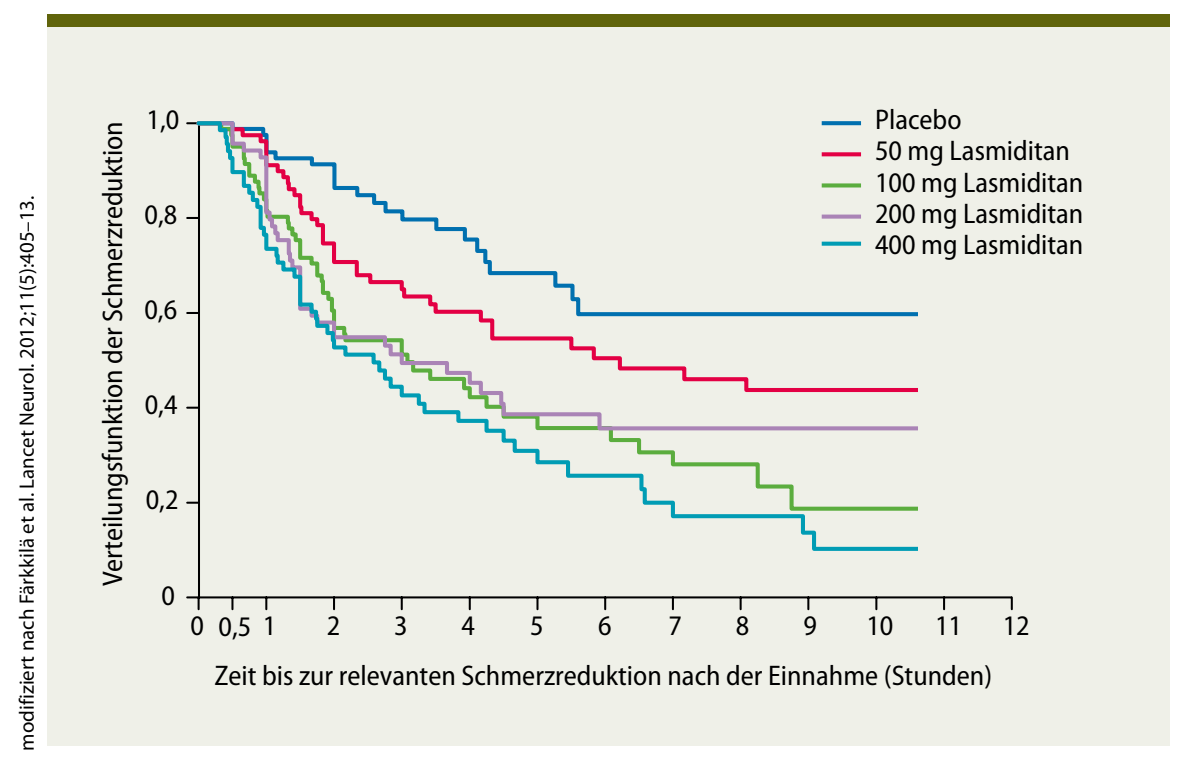

Abb. 1: Lasmiditan besserte dosisabhängig die Kopfschmerzen.

genschaften an Gefäßen der Dura und der Hirnbasis vermittelt und die Aktivierung dieser Rezeptoren bedingt einen Teil der Wirkung bei akuten Migräneattacken. Dieser Wirkmechanismus erklärt auch, warum Triptane bei vaskulären Erkrankungen wie transitorischen ischämischen Attacken (TIA), Schlaganfall, koronarer Herzerkrankung (KHK) und Zustand nach Myokardinfarkt kontraindiziert sind.

Ziel ist es daher, neue Substanzen zu entwickeln, die bei der Migräne wirksam sind und keine vasokonstriktiven Eigen- und mittelschwer auf leicht oder keine Kopfschmerzen nach zwei Stunden. Für die Studie wurden 512 Patienten rekrutiert, von denen 391 letztlich behandelt wurden. Die Patienten waren im Schnitt 40 Jahre alt und 85-90\% der Patienten waren Frauen. Etwa $60 \%$ hatten mittelschwere Attacken und rund $40 \%$ eine schwere Attacke.

Für den primären Endpunkt ergab sich eine lineare Beziehung zwischen der Besserung der Kopfschmerzen nach zwei Stunden und der Dosis von Lasmiditan (Abb. 1). Der Unterschied zu Placebo be- trug für $50 \mathrm{mg}$ Lasmiditan 17,9\%, für die 100-mg-Dosis 38,2\%, für die 200-mgDosis $28,8 \%$ und für die 400-mg-Dosis $38,7 \%$.

Auch die Nebenwirkungen waren dosisabhängig. Die Raten betrugen $65 \%$ für die 50-mg-Dosis, $52 \%$ für die 100-mgDosis, $86 \%$ für die 200-mg-Dosis und $84 \%$ für die 400-mg-Dosis. Die häufigsten Nebenwirkungen waren unsystematischer Schwindel, Müdigkeit, Drehschwindel, Parästhesien und Benommenheit.

Fazit: Lasmiditan bietet eine effektive Behandlung akuter Migräneattacken. Die Wirkung ist dosisabhängig.

Färkkilä M et al. for the COL MIG-202 study group. Efficacy and tolerability of lasmiditan, an oral $5-\mathrm{HT}(1 \mathrm{~F})$ receptor agonist, for the acute treatment of migraine: a phase 2 randomised, placebo-controlled, parallel-group, dose-ranging study. Lancet Neurol. 2012;11(5): 405-13.

Kommentar von Hans-Christoph Diener: Könnten die Ergebnisse dieser Studie in einer weiteren Studie repliziert werden, stünde mit Lasmiditan eine neue Substanz zur Behandlung akuter Migräneattacken zur Verfügung. Angesichts der relativ häufigen zentralen Nebenwirkungen wie Schwindel, Benommenheit, Müdigkeit und Parästhesien wird diese Substanz aber wahrscheinlich nur bei Patienten zum Einsatz kommen, bei denen Kontraindikationen gegen ein Triptan bestehen und die dann Willens sind, die entsprechenden Nebenwirkungen in Kauf zu nehmen.

Wahrscheinlich erklären sich die Nebenwirkungen durch die Expression von 5-HT1FRezeptoren im vestibulären Kerngebiet und im Zerebellum. Ob die Phase-II-Studie mit 100 oder 200 mg Lasmiditan oral durchgeführt werden wird, ist im Moment noch nicht bekannt.

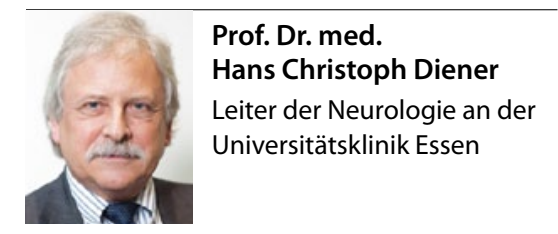

